\title{
Estimating the Parameters of the Generalized Exponential Distribution in Presence of Hybrid Censoring
}

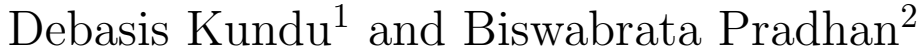

\begin{abstract}
The two most popular censoring schemes are type-I and type-II censoring schemes. Hybrid censoring scheme is a mixture of type-I and type-II censoring schemes. In this paper we mainly consider the analysis of hybrid censored data when the lifetime distribution of the individual item is a two-parameter generalized exponential distribution. It is observed that the maximum likelihood estimators can not be obtained in closed form. We propose to use the EM algorithm to compute the maximum likelihood estimators. We obtain the observed Fisher information matrix using the missing information principle and it can be used for constructing the asymptomatic confidence intervals. We also obtain the Bayes estimates of the unknown parameters under the assumption of independent gamma priors using the importance sampling procedure. One data set has been analyzed for illustrative purposes.
\end{abstract}

KEYwORDs: Maximum likelihood estimators; EM algorithm; asymptotic distribution; Fisher information matrix; Type-I censoring; Type-II censoring; Importance sampling.

${ }^{1}$ Department of Mathematics and Statistics, Indian Institute of Technology Kanpur, Pin 208016, INDIA. Corresponding Author: Phone: 91-512-2597141; Fax: 91-512-2597500; e-mail: kundu@iitk.ac.in

${ }^{2}$ SQC \& OR Unit, Indian Statistical Institute, 203 B.T. Road, Kolkata, Pin 700108, India

\section{INTRODUCTION}

The two most common censoring schemes are termed as type-I and type-II censoring schemes. Briefly, they can be described as follows; consider $n$ items under observation in a particular 
experiment. In the conventional type-I censoring scheme, the experiment continues up to a pre-specified time $T$. On the other hand the conventional type-II censoring scheme requires the experiment to continue until a pre-specified number of failures $R \leq n$ occur. A mixture of type-I and type-II censoring schemes is known as the hybrid censoring scheme and it can be described as follows. Suppose $n$ identical units are put to test under the same environmental conditions and the lifetime of each unit is independent and identically distributed (i.i.d.) random variables. The test is terminated when a pre-chosen number $R$, out of $n$ items have failed or a pre-determined time $T$, on test has been reached.

Therefore, under this censoring scheme we have one of the following two types of observations;

CASE I: $\left\{y_{1: n}<\ldots<y_{R: n}<T\right\}$

CASE II: $\left\{y_{1: n}<\ldots<y_{d: n}<T\right\}, \quad$ if $\quad 0 \leq d<R \quad$ and $\quad y_{d: n}<T<y_{d+1: n}$, here $y_{1: n}<y_{2: n}<\ldots$ denote the observed failure times of the experimental units. A schematic representation of the hybrid censoring scheme is provided in Figure 1.

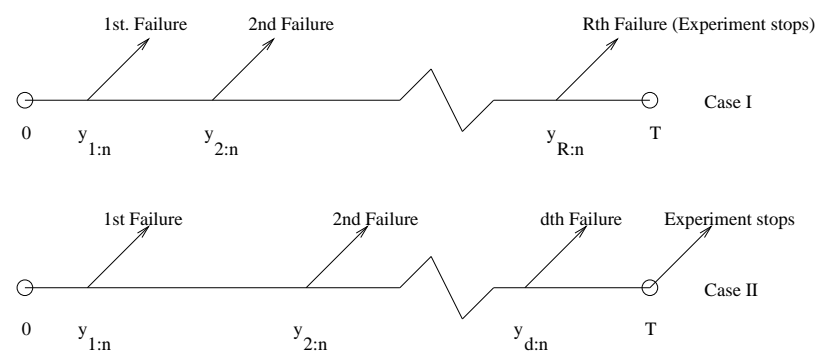

Figure 1: A schematic diagram for the hybrid censoring scheme.

Epstein [8] first introduced the hybrid censoring and it has been used in reliability acceptance test in MIL-STD-781C [19]. Epstein [8] analyzed the data under the assumption of exponential lifetimes of the experimental units. Epstein [8] also proposed a two-sided confidence intervals of the mean lifetime without any formal proof. Some modifications of Epstein's proposition were suggested by Fairbanks et al. [9]. Chen and Bhattacharya [2] 
provided the exact confidence interval of the exponential parameter based on the exact conditional maximum likelihood estimate (MLE) obtained by inverting the conditional moment generating function (MGF). Some simplifications of the the exact distribution have been suggested by Childs et al. [4]. Draper and Guttman [5] provided the Bayesian estimates and the highest posterior density (HPD) credible interval based on the gamma prior. Gupta and Kundu [10] compared different methods using Monte Carlo simulations. For some of the related references, the readers are referred to Ebrahimi [6, 7], Jeong, Park and Yum [14], Kundu [15], Banerjee and Kundu [1] and the references cited therein.

In this paper we consider the analysis of the hybrid censored lifetime data when the lifetime of each experimental unit follows a two-parameter generalized exponential (GE) distribution. The two-parameter GE was originally proposed by Gupta and Kundu [11] and it has received considerable attention in the recent years because of its flexibility and wide scale applicability. See for example, the review article by Gupta and Kundu [13] on recent development of the GE distribution. Two-parameter GE distribution with the shape parameter $\alpha>0$ and scale parameter $\lambda>0$ has the probability density function (PDF) for $x>0$ as;

$$
f_{G E}(x ; \alpha, \lambda)=\alpha \lambda e^{-\lambda x}\left(1-e^{-\lambda x}\right)^{\alpha-1}
$$

From now on a two-parameter GE distribution with the PDF (1) will be denoted by GE $(\alpha, \lambda)$ and the corresponding CDF will be denoted by $F_{G E}(x ; \alpha, \lambda)$.

The aim of this paper is two fold. First we consider the point and interval estimates of the unknown parameters based on the frequentist approach. It is observed that the MLEs of the unknown parameters can not be obtained in closed form. We propose to use the EM algorithm similarly as in $\mathrm{Ng}$ et al. [20], to compute the MLEs. Using the missing information principle we calculate the observed Fisher information matrix, which can be used for constructing the asymptotic confidence intervals of the unknown parameters. The 
second aim of this paper is to consider the Bayesian inference for the unknown parameters when the data are hybrid censored. The Bayes estimates can not be obtained in closed form. Using the importance sampling procedure we obtain the Bayes estimates and also the HPD credible intervals under the assumptions of independent gamma priors of both the shape and scale parameters. We have used one data set for illustrative purpose.

Rest of the paper is organized as follows. In section 2, we provide the EM algorithm. The Fisher information matrix is provided in section 3. Bayesian inferences are presented in section 4. Analysis of one data set and discussions appear in section 5.

\section{EM Algorithm}

Based on the observed data, ignoring the additive constant, the log-likelihood function for Case I and Case II can be written as

$L(\alpha, \lambda \mid d a t a)=d \ln \alpha+d \ln \lambda-\lambda \sum_{i=1}^{d} y_{i: n}+(\alpha-1) \sum_{i=1}^{d} \ln \left(1-e^{-\lambda y_{i: n}}\right)+(n-d) \ln \left(1-\left(1-e^{-\lambda c}\right)^{\alpha}\right)$.

Note that for Case I, $d=R$ and $c=y_{R: n}$, and for Case II, $0 \leq d \leq R-1$ and $c=T$.

When $d=0, L(\alpha, \lambda \mid$ data $)=n \ln \left(1-\left(1-e^{-\lambda c}\right)^{\alpha}\right)$, which can be made arbitrary small by choosing $\lambda \rightarrow 0$, for any fixed $\alpha$. It implies that the MLEs of $\alpha$ and $\lambda$ do not exist when $d=0$. So for computing the MLEs, it is assumed that $d>0$.

Note that the explicit solutions of the two normal equations can not be obtained. We propose to use the EM algorithm to compute the MLEs of the unknown parameters, treating it as a missing value problem. Let us denote the observed and the censored data by $Y=$ $\left(Y_{1: n}, \ldots, Y_{d: n}\right)$ and $Z=\left(Z_{1}, \ldots, Z_{n-d}\right)$ respectively. Here for a given $d, Z_{1}, \ldots, Z_{n-d}$ are not observable. The censored data vector $Z$ can be thought of as missing data. The combination of $W=(Y, Z)$ forms the complete data set. If we denote the log-likelihood function of the 
uncensored data set by $L_{c}(W ; \alpha, \lambda)$ then ignoring the additive constant;

$$
\begin{aligned}
L_{c}(W ; \alpha, \lambda)= & n \ln \alpha+n \ln \lambda-\lambda\left(\sum_{i=1}^{d} y_{i: n}+\sum_{i=1}^{n-d} z_{i}\right) \\
& +(\alpha-1)\left(\sum_{i=1}^{d} \ln \left(1-e^{-\lambda y_{i: n}}\right)+\sum_{i=1}^{n-d} \ln \left(1-e^{-\lambda z_{i}}\right)\right) .
\end{aligned}
$$

For the 'E'-step of the EM algorithm, one needs to compute the pseudo log-likelihood function as $L_{s}(\alpha, \lambda \mid$ data $)=E\left(L_{c}(W ; \alpha, \lambda) \mid Y\right)$. Therefore,

$$
\begin{aligned}
L_{s}(\alpha, \lambda \mid \text { data })= & n \ln \alpha+n \ln \lambda-\lambda \sum_{i=1}^{d} y_{i: n}+(\alpha-1) \sum_{i=1}^{d} \ln \left(1-e^{-\lambda y_{i: n}}\right) \\
& -\lambda \sum_{i=1}^{n-d} E\left(Z_{i} \mid Z_{i}>c\right)+(\alpha-1) \sum_{i=1}^{n-d} E\left(\ln \left(1-e^{-\lambda Z_{i}}\right) \mid Z_{i}>c\right)
\end{aligned}
$$

For further development we need the following results. The proofs can be obtained similarly as in $\mathrm{Ng}$ et al. [20].

$\operatorname{RESUlT} 1:$ Given $Y_{1: n}=y_{1: n}, \ldots, Y_{R: n}=y_{R: n}$, the conditional PDF of $Z_{j}$, for $j=1, \ldots, n-R$ is

$$
f_{Z \mid Y}\left(z_{j} \mid Y_{1: n}=y_{1: n}, \ldots, Y_{R: n}=y_{R: n}\right)=\frac{f_{G E}\left(z_{j} ; \alpha, \lambda\right)}{1-F_{G E}\left(y_{R: n} ; \alpha, \lambda\right)} ; \quad z_{j}>y_{R: n}
$$

and $Z_{j}$ and $Z_{k}$ for $j \neq k$ are conditionally independent.

REsult 2: Given $d$ and $Y_{1: n}=y_{1: n}, \ldots, Y_{d: n}=y_{d: n}<T$, the conditional PDF of $Z_{j}$, for $j=1, \ldots, n-d$ is

$$
f_{Z \mid Y}\left(z_{j} \mid Y_{1: n}=y_{1: n}, \ldots, Y_{d: n}=y_{d: n}<T\right)=\frac{f_{G E}\left(z_{j} ; \alpha, \lambda\right)}{1-F_{G E}(T ; \alpha, \lambda)} ; \quad z_{j}>T
$$

and $Z_{j}$ and $Z_{k}$ for $j \neq k$ are conditionally independent.

Note that we can write;

$$
\begin{aligned}
A(c, \alpha, \lambda)=E\left(Z_{j} \mid Z_{j}>c\right) & =\frac{\alpha \lambda}{1-F_{G E}(c ; \alpha, \lambda)} \times \int_{c}^{\infty} x e^{-\lambda x}\left(1-e^{-\lambda x}\right)^{\alpha-1} d x \\
& =-\frac{\alpha}{\lambda\left(1-F_{G E}(c ; \alpha, \lambda)\right)} u(\lambda c, \alpha),
\end{aligned}
$$


where

$$
\begin{aligned}
u(a, \alpha)=\int_{0}^{e^{-a}}(1-z)^{\alpha-1} \ln z d z . \\
B(c, \alpha, \lambda)=E\left(\ln \left(1-e^{-\lambda Z_{j}}\right) \mid Z_{j}>c\right) \\
=\frac{\alpha \lambda}{1-F(c ; \alpha, \lambda)} \times \int_{c}^{\infty} e^{-\lambda x}\left(1-e^{-\lambda x}\right)^{\alpha-1} \ln \left(1-e^{-\lambda x}\right)^{\alpha-1} d x \\
=\frac{1}{\alpha(1-F(c ; \alpha, \lambda))} \int_{\left(1-e^{-c \lambda}\right)^{\alpha}}^{1} \ln y d y \\
=\frac{1}{\alpha(1-F(c ; \alpha, \lambda))}\left[\left(1-e^{-c \lambda}\right)^{\alpha}\left(1-\alpha \ln \left(1-e^{-c \lambda}\right)\right)-1\right] .
\end{aligned}
$$

Now the 'M'-step involves the maximization of the pseudo log-likelihood function (4). Therefore, if at the $k$-th stage the estimate of $(\alpha, \lambda)$ is $\left(\alpha^{(k)}, \lambda^{(k)}\right)$, then $\left(\alpha^{(k+1)}, \lambda^{(k+1)}\right)$ can be obtained by maximizing

$$
\begin{aligned}
g(\alpha, \lambda)= & n \ln \alpha+n \ln \lambda-\lambda \sum_{i=1}^{d} y_{i: n}+(\alpha-1) \sum_{i=1}^{d} \ln \left(1-e^{-\lambda y_{i: n}}\right) \\
& -\lambda(n-d) A\left(c, \alpha^{(k)}, \lambda^{(k)}\right)+(\alpha-1)(n-d) B\left(c, \alpha^{(k)}, \lambda^{(k)}\right) .
\end{aligned}
$$

The maximization of (9) can be performed by using similar technique as of Gupta and Kundu [12]. First find $\lambda^{(k+1)}$ by solving a fixed point type equation as

$$
h(\lambda)=\lambda
$$

The function $h(\lambda)$ is defined as follows;

$$
h(\lambda)=\left[\frac{1}{n} \sum_{i=1}^{d} y_{i: n}+\frac{n-d}{n} A-\frac{1}{n}(\widehat{\alpha}(\lambda)-1) \sum_{i=1}^{d} \frac{y_{i: n} e^{-\lambda y_{i: n}}}{1-e^{-\lambda y_{i: n}}}\right]^{-1}
$$

where

$$
A=A\left(c, \alpha^{(k)}, \lambda^{(k)}\right), \quad B=B\left(c, \alpha^{(k)}, \lambda^{(k)}\right), \quad \text { and } \quad \widehat{\alpha}(\lambda)=-\frac{n}{\sum_{i=1}^{d} \ln \left(1-e^{-\lambda y_{i: n}}\right)+(n-d) B} .
$$

Once $\lambda^{(k+1)}$ is obtained, $\alpha^{(k+1)}$ is obtained as $\alpha^{(k+1)}=\widehat{\alpha}\left(\lambda^{(k+1)}\right)$. 


\section{Fisher Information Matrices}

In this section we present the observed Fisher information matrix obtained using the missing value principles of Louis [18]. The observed Fisher information matrix can be used to construct the asymptotic confidence intervals. The idea of missing information principle is as follows;

$$
\text { Observed information }=\text { Complete information }- \text { Missing information }
$$

Let us use the following notation; $\theta=(\alpha, \lambda), Y=$ the observed vector, $W=$ the complete data, $I_{W}(\theta)=$ the complete information, $I_{Y}(\theta)=$ the observed information, $I_{W \mid Y}=$ the missing information. Then (11) can be expressed as

$$
I_{Y}(\theta)=I_{W}(\theta)-I_{W \mid Y}(\theta)
$$

The complete information $I_{W}(\theta)$ is given by

$$
I_{W}(\theta)=-E\left[\frac{\partial^{2} L_{c}(W ; \theta)}{\partial \theta^{2}}\right]
$$

The Fisher information matrix of the censored observations can be written as

$$
I_{W \mid Y}(\theta)=-(n-d) \quad E_{Z \mid Y}\left[\frac{\partial^{2} \ln f_{Z}(z \mid Y, \theta)}{\partial \theta^{2}}\right] .
$$

So we obtain the observed information as

$$
I_{Y}(\theta)=I_{W}(\theta)-I_{W \mid Y}(\theta)
$$

and naturally, the asymptotic variance covariance matrix of $\widehat{\theta}$ can be obtained by inverting $I_{Y}(\widehat{\theta})$.

Note that $I_{W}(\theta)$ and $I_{W \mid Y}(\theta)$ are both of the order $2 \times 2$. We present all the elements of both the matrices. The elements of the matrix $I_{W}(\theta)$ for complete data set are already 
available in Gupta and Kundu [12]. We report it here for completeness purpose. If the $(i, j)$-th element of the matrix $I_{W}(\theta)$ is denoted by $a_{i j}(\alpha, \lambda)$, they they are as follows;

$$
\begin{aligned}
& a_{11}=\frac{n}{\alpha^{2}} \\
& a_{22}=\frac{n}{\lambda^{2}}+\frac{n \alpha(\alpha-1)}{\lambda^{2}} \int_{0}^{\infty} x^{2} e^{-2 x}\left(1-e^{-x}\right)^{\alpha-3} d x . \\
& a_{12}=a_{21}=-\frac{n \alpha}{\lambda} \int_{0}^{\infty} x e^{-2 x}\left(1-e^{-x}\right)^{\alpha-2} d x .
\end{aligned}
$$

Now we present $I_{W \mid Y}(\theta)$. If

$$
I_{W \mid Y}(\theta)=(n-d)\left[\begin{array}{ll}
b_{11}(c ; \alpha, \lambda) & b_{12}(c ; \alpha, \lambda) \\
b_{21}(c ; \alpha, \lambda) & b_{22}(c ; \alpha, \lambda)
\end{array}\right],
$$

then

$$
\begin{aligned}
b_{11}(c ; \alpha, \lambda) & =\frac{1}{\alpha^{2}}-\left[\ln \left(1-e^{-\lambda c}\right)\right]^{2} \frac{\left(1-e^{-\lambda c}\right)^{\alpha}}{\left(1-\left(1-e^{-\lambda c}\right)^{\alpha}\right)^{2}}, \\
b_{22}(c ; \alpha, \lambda) & =\frac{1}{\lambda^{2}}+(\alpha-1) h_{1}(c ; \alpha, \lambda)-\frac{\alpha c^{2} e^{-\lambda c}\left(1-e^{-c \lambda}\right)^{\alpha-2}\left(\alpha e^{-c \lambda}-1+\left(1-e^{-c \lambda}\right)^{\alpha}\right)}{\left(1-\left(1-e^{-c \lambda}\right)^{\alpha}\right)^{2}}, \\
b_{12}(c ; \alpha, \lambda) & =-h_{2}(c ; \alpha, \lambda)+\frac{c e^{-c \lambda}\left(1-e^{-c \lambda}\right)^{\alpha-1}\left(1+\alpha \ln \left(1-e^{-c \lambda}\right)-\left(1-e^{-c \lambda}\right)^{\alpha}\right)}{\left(1-\left(1-e^{-c \lambda}\right)^{\alpha}\right)^{2}} \\
& =b_{21}(c ; \alpha, \lambda),
\end{aligned}
$$

where

$$
\begin{aligned}
& h_{1}(c ; \alpha, \lambda)=\frac{1}{\lambda^{2}\left(1-\left(1-e^{-c \lambda}\right)^{\alpha}\right)} \times \int_{\left(1-e^{-c \lambda}\right)^{\alpha}}^{1}\left(\ln \left(1-u^{1 / \alpha}\right)\right)^{2}\left(1-u^{1 / \alpha}\right) u^{-2 / \alpha} d u \\
& h_{2}(c ; \alpha, \lambda)=\frac{1}{\lambda\left(1-\left(1-e^{-c \lambda}\right)^{\alpha}\right)} \times \int_{\left(1-e^{-c \lambda}\right)^{\alpha}}^{1}\left(-\ln \left(1-u^{1 / \alpha}\right)\right)\left(1-u^{1 / \alpha}\right) u^{-1 / \alpha} d u .
\end{aligned}
$$

\section{Bayes Estimates}

In this section we consider the Bayes estimates of the unknown parameters. Unfortunately, when both the parameters are unknown then there does not exist any natural conjugate 
priors. In this paper similarly as in Raqab and Madi [21] or Kundu and Gupta [16], it is assumed that $\alpha$ and $\lambda$ have the following independent gamma priors;

$$
\begin{aligned}
& \pi_{1}(\alpha) \propto \alpha^{a_{1}-1} e^{-b_{1} \alpha}, \quad \alpha>0, \\
& \pi_{2}(\lambda) \propto \lambda^{a_{2}-1} e^{-b_{2} \lambda}, \quad \lambda>0 .
\end{aligned}
$$

Here all the hyper parameters $a_{1}, b_{1}, a_{2}, b_{2}$ are assumed to be known and non-negative.

Based on the observed sample $\left\{y_{1: n}, \ldots, y_{d: n}\right\}$, from the hybrid censoring scheme the likelihood function becomes;

$$
l(\text { data } \mid \alpha, \lambda) \propto \alpha^{d} \lambda^{d} e^{-\lambda \sum_{i=1}^{d} y_{i: n}} e^{(\alpha-1) \sum_{i=1}^{d} \ln \left(1-e^{\left.-\lambda y_{i: n}\right)}\right.} e^{(n-d) \ln \left(1-\left(1-e^{-\lambda c}\right)^{\alpha}\right)} .
$$

The joint posterior density function of $\alpha$ and $\lambda$ can be written as

$$
\begin{aligned}
\pi(\alpha, \lambda \mid \text { data }) & =\frac{l(\text { data } \mid \alpha, \lambda) \pi_{1}(\alpha) \pi_{2}(\lambda)}{\int_{0}^{\infty} \int_{0}^{\infty} l(\text { data } \mid \alpha, \lambda) \pi_{1}(\alpha) \pi_{2}(\lambda) d \alpha d \lambda} . \\
\propto & \alpha^{a_{1}+d-1} e^{-\alpha\left(b_{1}-\sum_{i=1}^{d} \ln \left(1-e^{-\lambda y_{i: n}}\right)\right)} \quad \lambda^{a_{2}+d-1} e^{-\lambda\left(b_{2}+\sum_{i=1}^{d} y_{i: n}\right)} \times \\
& e^{\left.-\sum_{i=1}^{d} \ln \left(1-e^{-\lambda y_{i: n}}\right)+(n-d) \ln \left(1-\left(1-e^{-\lambda c}\right)^{\alpha}\right)\right)}
\end{aligned}
$$

Therefore, the Bayes estimate of any function of $\alpha$ and $\lambda$, say $\theta(\alpha, \lambda)$ under the squared error loss function is;

$$
\widehat{\theta}_{B}=E_{\alpha, \lambda \mid d a t a}(\theta(\alpha, \lambda))=\frac{\int_{0}^{\infty} \int_{0}^{\infty} \theta(\alpha, \lambda) l(\text { data } \mid \alpha, \lambda) \pi_{1}(\alpha) \pi_{2}(\lambda) d \alpha d \lambda}{\int_{0}^{\infty} \int_{0}^{\infty} l(\text { data } \mid \alpha, \lambda) \pi_{1}(\alpha) \pi_{2}(\lambda) d \alpha d \lambda}
$$

It is not possible to compute (17) analytically in this case. We will provide a simple importance sampling procedure to compute the point estimate of any function of $\alpha$ and $\lambda$, similarly as in Raqab and Madi [21]. Using the idea of Chen and Shao [3] we obtain its HPD credible interval also.

Note that the joint posterior density function of $\alpha$ and $\lambda(16)$ can be written as

$$
\pi(\alpha, \lambda \mid d a t a) \propto g_{\lambda}\left(a_{2}^{*}, b_{2}^{*}\right) \times g_{\alpha \mid \lambda}\left(a_{1}^{*}, b_{1}^{*}\right) \times g_{3}(\alpha, \lambda),
$$


where $g_{\alpha \mid \lambda}\left(a_{1}^{*}, b_{1}^{*}\right)$ is a gamma density function with the shape and scale parameter as $a_{1}^{*}=$ $a_{1}+d$ and $b_{1}^{*}=b_{1}-\sum_{i=1}^{d} \ln \left(1-e^{-\lambda y_{i: n}}\right)$ respectively. Similarly, $g_{\lambda}\left(a_{2}^{*}, b_{2}^{*}\right)$ is a gamma density function with the shape and scale parameter as $a_{2}^{*}=a_{2}+d$ and $b_{2}^{*}=b_{2}+\sum_{i=1}^{d} y_{i: n}$ respectively. Moreover,

$$
g_{3}(\alpha, \lambda)=\frac{1}{\left(b_{1}-\sum_{i=1}^{d} \ln \left(1-e^{-\lambda y_{i: n}}\right)\right)^{a_{1}+d}} e^{(n-d) \ln \left(1-\left(1-e^{-\lambda c}\right)^{\alpha}\right)-\sum_{i=1}^{d} \ln \left(1-e^{\left.-\lambda y_{i: n}\right)},\right.}
$$

is a function of $\alpha$ and $\lambda$. The PDF of a gamma density function with the shape and scale parameters as $a$ and $b$ respectively is

$$
f(x ; a, b)=\frac{b^{a}}{\Gamma(a)} x^{a-1} e^{-b x} ; \quad x>0,
$$

and it will be denoted by gamma $(a, b)$.

Similarly as in Raqab and Madi [21], it is quite easy to obtain a simulation consistent estimator of $\widehat{\theta}_{B}$ using the importance sampling scheme as follows;

- Step 1: Generate $\lambda_{1}$ from $g_{\lambda}\left(a_{2}^{*}, b_{2}^{*}\right) \sim \operatorname{gamma}\left(a_{2}+d, b_{2}+\sum_{i=1}^{d} y_{i: n}\right)$

- Step 2: Generate $\alpha_{1}$ from $g_{\alpha \mid \lambda}\left(a_{1}^{*}, b_{1}^{*}\right) \sim \operatorname{gamma}\left(a_{1}+d, b_{1}-\sum_{i=1}^{d} \ln \left(1-e^{-\lambda_{1} y_{i: n}}\right)\right)$

- Step 3: Repeat Step 1 and Step 2, $N$ times and obtain $\left(\alpha_{1}, \lambda_{1}\right), \ldots,\left(\alpha_{N}, \lambda_{N}\right)$.

- Step 4: The Bayes estimate of $\theta$ under squared error loss function can be approximated

$$
\widehat{\theta}_{B} \approx \frac{\frac{1}{N} \sum_{i=1}^{N} \theta\left(\alpha_{i}, \lambda_{i}\right) g_{3}\left(\alpha_{i}, \lambda_{i}\right)}{\frac{1}{N} \sum_{i=1}^{N} g_{3}\left(\alpha_{i}, \lambda_{i}\right)} .
$$

Now we obtain the credible interval of $\theta$. Let us denote $\pi(\theta \mid$ data $)$ and $\Pi(\theta \mid$ data $)$ as the posterior density function and posterior distribution function of $\theta$ respectively. Also let $\theta^{(\beta)}$ be the $\beta$-th quantile of $\theta$, i.e.

$$
\theta^{(\beta)}=\inf \{\theta: \Pi(\theta \mid \text { data }) \geq \beta),
$$


where $0<\beta<1$. Observe that for a given $\theta^{*}$,

$$
\Pi\left(\theta^{*} \mid \text { data }\right)=E\left(1_{\theta \leq \theta^{*}} \mid \text { data }\right)
$$

where $1_{\theta \leq \theta^{*}}$ is the indicator function. Then a simulation consistent estimator of $\Pi\left(\theta^{*} \mid\right.$ data $)$ can be obtained as

$$
\Pi\left(\theta^{*} \mid \text { data }\right)=\frac{\frac{1}{N} \sum_{i=1}^{N} 1_{\theta \leq \theta^{*}} g_{3}\left(\alpha_{i}, \lambda_{i}\right)}{\frac{1}{N} \sum_{i=1}^{N} g_{3}\left(\alpha_{i}, \lambda_{i}\right)} .
$$

Let $\left\{\theta_{(i)}\right\}$ be the ordered value of $\left\{\theta_{i}\right\}$, and denote

$$
w_{i}=\frac{g_{3}\left(\alpha_{(i)}, \lambda_{(i)}\right)}{\sum_{i=1}^{N} g_{3}\left(\alpha_{(i)}, \lambda_{(i)}\right)}
$$

for $i=1 \ldots, N$. Then we have

$$
\Pi\left(\theta^{*} \mid \text { data }\right)=\left\{\begin{array}{ccc}
0 & \text { if } & \theta^{*}<\theta_{(1)} \\
\sum_{j=1}^{i} w_{j} & \text { if } & \theta_{(i)} \leq \theta^{*}<\theta_{(i+1)} \\
1 & \text { if } & \theta^{*} \geq \theta_{(n)}
\end{array}\right.
$$

Therefore, $\theta^{(\beta)}$ can be approximated by

$$
\widehat{\theta}^{(\beta)}=\left\{\begin{array}{ccc}
\theta_{(1)} & \text { if } & \beta=0 \\
\theta_{(i)} & \text { if } & \sum_{j=1}^{i-1} w_{j}<\beta \leq \sum_{j=1}^{i} w_{j}
\end{array}\right.
$$

To obtain a $100(1-\beta) \%$ HPD credible interval for $\theta$, let $R_{j}=\left(\widehat{\theta}^{\left(\frac{j}{N}\right)}, \widehat{\theta}^{\left(\frac{j+(1-\beta) N}{N}\right)}\right)$ for $j=1, \ldots,[\beta N]$, here $[a]$ denotes the largest integer less than or equal to $a$. Then choose $R_{j^{*}}$ among all the $R_{j}$ 's such that it has the smallest width.

\section{Data Analysis and Discussions}

In this section we perform the following data analysis for illustrative purpose. The data set is from Lawless [17] ( page 228). The data given here arose in tests on endurance of deep groove ball bearings. The data are the numbers of million revolution before failure for each of the 23 ball bearings in the life test and they are: 17.88, 28.92, 33.00, 41.52, 
$42.12,45.60,48.80,51.84,51.96,54.12,55.56,67.80,68.64,68.64,68.88,84.12,93.12,98.64$, 105.12, 105.84, 127.92, 128.04 and 173.40. It has been analyzed by several authors. It has been observed by Gupta and Kundu [12] that the two-parameter GE distribution can be used quite effectively to analyze this data set.

We have created two artificially hybrid censored data sets from the above uncensored data set, using the following censoring schemes: Scheme 1: $R=20, T=100$ and Scheme 2: $R=$ $15, T=75$. In both the cases we have estimated the unknown parameters using the MLEs and the Bayes estimates. For computing the MLEs we have used the EM algorithm as described in Section 2 and also computed the 95\% confidence intervals using the observed Fisher information matrix as provided in Section 3. For computing the Bayes estimates we have mainly considered the squared error loss function in both the cases. For comparison purposes (with the MLEs), we have mainly assumed the non-informative priors i.e. $a_{1}=b_{1}=a_{2}=b_{2}$ $=0.0$. The Bayes estimates in all the cases are obtained by using importance samples of size $\mathrm{N}=10,000$.

For Scheme 1, the MLEs of $\alpha$ and $\lambda$ are 4.9892 and 0.0311 respectively. The corresponding $95 \%$ confidence intervals are $(2.4767,7.5018)$ and $(0.0159,0.0461)$ respectively. Similarly, the Bayes estimates of $\alpha$ and $\lambda$ are 4.2083 and 0.0281 respectively and the corresponding $95 \%$ HPD credible intervals are $(2.1642,7.1195)$ and $(0.0185,0.0390)$. Interestingly, in both the cases the asymptotic confidence intervals are slightly larger than the HPD credible intervals obtained by using the non-informative priors. Although it appears that the MLEs and Bayes estimates are quite different but if we consider the corresponding estimated distribution functions they match quite well, see Figure 2.

For Scheme 2, the MLEs of $\alpha$ and $\lambda$ are 7.1503 and 0.0393, the corresponding Bayes estimates are 5.3981 and 0.0330 respectively. The $95 \%$ asymptotic confidence intervals and credible intervals for $\alpha$ and $\lambda$ are $(3.6674,10.6332),(0.0214,0.0571)$ and $(2.6403,8.4319)$, 




Figure 2: The fitted distribution functions based on MLEs and Bayes estimators for Scheme 1.

$(0.0219,0.0458)$ respectively. We have plotted the fitted distribution functions based on MLEs and Bayes estimators. It is clear from the picture that the distance between the two distribution functions is more for Scheme 2 than Scheme 1. Although the MLEs and Bayes estimates under the assumption of non-informative priors are close to each other when the censoring is small, but when the censoring proportion is large then they can be quite different.

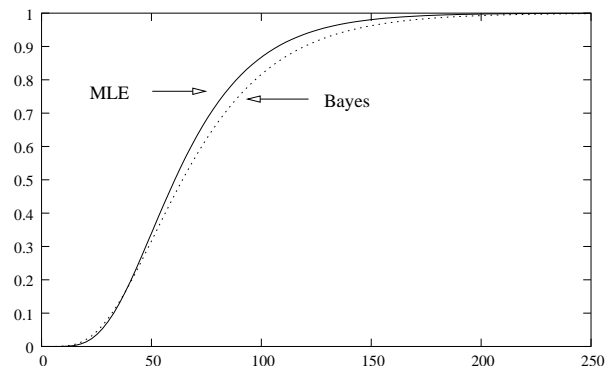

Figure 3: The fitted distribution functions based on MLEs and Bayes estimators for Scheme 2.

Now we want to see the effect of the hyper parameters on the Bayes estimates and also on HPD credible intervals. We have taken the following informative priors $a_{1}=3.0, b_{1}=1.0, a_{2}$ $=0.01$ and $b_{2}=1.0$. Based on the above hyper parameters, for Scheme 1, the Bayes estimates of $\alpha$ and $\lambda$ are 4.0339 and 0.0276 respectively. The corresponding 95\% HPD credible intervals for $\alpha$ and $\lambda$ are $(2.1758,6.6247)$ and $(0.0184,0.0367)$ respectively. Similarly for Scheme 2, 
the Bayes estimates of $\alpha$ and $\lambda$ are 4.7286 and 0.0313 and the corresponding 95\% HPD credible intervals are $(2.4441,8.2670)$ and $(0.0207,0.0483)$ respectively. Therefore, it is clear that the Bayes estimates are quite robust, they do not depend on the hyper parameters very much. Although it may be observed that the length of the HPD credible intervals based on informative priors are slightly smaller than the corresponding length of the HPD credible intervals based on non-informative priors, as expected. Therefore, the prior information should be used if they are available.

Finally we should mention that our method can be used for other censoring plans also, for example type-I, type-II, Type-II hybrid (see for example Childs et al. [4] or progressive censoring plans. More work is needed in these directions.

\section{ACKNOWLEDGEMENTS}

The authors would like to thank the associate editor and two referees for their valuable comments.

\section{References}

[1] Banerjee, A. and Kundu, D. (2008), "Inference based on Type-II hybrid censored data from Weibull distribution", IEEE Transactions on Reliability, in press.

[2] Chen, S. and Bhattacharya, G. K. (1998), "Exact confidence bounds for an exponential parameter under hybrid censoring", Communications in Statistics - Theory and Methods, vol 17, $1857-1870$.

[3] Chen, Ming-Hui and Shao, Qi-Man (1999), "Monte Carlo estimation of Bayesian credible and HPD intervals", Journal of Computational and Graphical Statistics, vol. 8, 69-92. 
[4] Childs, A., Chandrasekhar, B., Balakrishnan, N., Kundu, D. (2003), "Exact likelihood inference based on type-I and type-II hybrid censored samples from the exponential distribution", Annals of the Institute of Statistical Mathematics, vol. 55, 319 - 330.

[5] Draper, N. and Guttman, I. (1987), "Bayesian analysis of hybrid life tests with exponential failure times", Annals of the Institute of Statistical Mathematics, vol. 39, 219 225.

[6] Ebrahimi, N. (1990), "Estimating the parameter of an exponential distribution from hybrid life test", Journal of Statistical Planning and Inference, 23, 255-261.

[7] Ebrahimi, N. (1992), "Prediction intervals for future failures in exponential distribution under hybrid censoring", IEEE Transactions on Reliability, vol. 41, 127 - 132.

[8] Epstein, B. (1954), "Truncated life tests in the exponential case", Annals of Mathematical Statistics, vol. 25, $555-564$.

[9] Fairbanks, K., Madison, R. and Dykstra (1982), "A confidence interval for an exponential parameter from a hybrid life test", Journal of the American Statistical Association, vol. $77,137-140$.

[10] Gupta, R. D. and Kundu, D. (1998), "Hybrid censoring schemes with exponential failure distribution", Communications in Statistics - Theory and Methods, vol. 27, 3065 - 3083.

[11] Gupta, R.D. and Kundu, D. (1999), "Generalized exponential distributions", Australian and New Zealand Journal of Statistics, vol. 41, 173-188.

[12] Gupta, R. D. and Kundu, D. (2001) "Exponentiated Exponential Family; An Alternative to Gamma and Weibull", Biometrical Journal, vol. 33, 117-130, 2001. 
[13] Gupta, R. D. and Kundu, D. (2007), "Generalized exponential distribution: existing methods and recent developments", Journal of the Statistical Planning and Inference, vol. $137,3537-3547$.

[14] Jeong, H.S., Park, J.I. and Yum, B.J. (1996), "Development of (r, T) hybrid sampling plans for exponential lifetime distributions", Journal of Applied Statistics, vol. 23, 601 607.

[15] Kundu, D. (2007), "On hybrid censored Weibull distribution", Journal of Statistical Planning and Inference, vol. 137, 2127-2142.

[16] Kundu, D. and Gupta, R.D. (2008), "Generalized exponential distribution; Bayesian Inference", Computational Statistics and Data Analysis, vol 52, 1873-1883.

[17] Lawless, J.F. (1982), Statistical models and methods for lifetime data, John Wiley and Sons, New York.

[18] Louis, T.A. (1982), "Finding the observed information matrix using the EM algorithm", Journal of the Royal Statistical Society, Ser B, 44, 226 - 233.

[19] MIL-STD-781- C (1977), Reliability Design Qualifications and Production Acceptance Test, Exponential Distribution, U.S. Government Printing Office, Washington, D.C.

[20] Ng, T., Chan, C.S. and Balakrishnan, N. (2002), "Estimation of parameters from progressively censored data using EM algorithm", Computational Statistics and Data Analysis, vol. $39,371-386$.

[21] Raqab, M.Z. and Madi, M.T. (2005), "Bayesian inference for the generalized exponential distribution", Journal of Statistical Computation and Simulation, vol. 75, no. 10, 841 852. 